\title{
Discrete hysteretic sine-Gordon model: soliton versus hysteresis
}

\author{
Peter A. Meleshenko ${ }^{1,2, *}$, Akim V. Tolkachev ${ }^{1}$, Mikhail E. Semenov1,2,3,4,**, Alla V. Perova ${ }^{3}$, Andrey I. Barsukov ${ }^{3}$, and \\ Alexander $F$. Klinskikh ${ }^{1}$ \\ ${ }^{1}$ Voronezh State University, Universitetskaya sq. 1, 394006, Voronezh, Russia \\ ${ }^{2}$ Zhukovsky-Gagarin Air Force Academy, Starykh Bolshevikov st. 54 "A", 394064, Voronezh, Russia \\ ${ }^{3}$ Voronezh State Technical University, XX-letiya Oktyabrya st. 84, 394006 Voronezh, Russia \\ ${ }^{4}$ Geophysical Survey of Russia Academy of Sciences, Lenina av. 189, 249035 Obninsk, Russia
}

\begin{abstract}
In this work we consider a nonlinear dynamical system which is a set of nonlinear oscillators coupled by springs with hysteretic blocks (hysteretic sine-Gordon model). The hysteretic nonlinearity is modeled using the phenomenological Bouc-Wen model). The wave processes (solitonic solutions) in such a system are investigated taking into account the hysteretic nature of the coupling.
\end{abstract}

\section{Introduction}

The theory of oscillations, which studies oscillations occurring in various systems, is an intensively developing field of modern mathematics and physics [1-4]. The main models of the theory of oscillations are the linear and nonlinear oscillators, rotators, RLC circuit, etc. These are used in modeling of physical processes in various real-life systems.

The standard model of wave processes is a finite and infinite chain of coupled (interacting) oscillators. Such chains are often used in radio engineering as filters that allocate or suppress signals with frequencies lying in a certain band. From the fundamental point of view, chains of oscillators are used as models of solid media with oscillations and waves with various properties [5-7]. Wave phenomena are widespread in nature: waves on the surface of a fluid, sound waves in a gas, compression-expansion waves in a solid, vibrations of a string and membrane, electromagnetic waves, etc.

In addition to nonlinear oscillations, there are also nonlinear waves the behaviour of which is described by nonlinear partial differential equations. Within the framework of the theory of nonlinear waves there exist the standard models, similar to the reference models in the theory of oscillations, namely, simple waves, shock waves, as well as the solitary waves (solitons), that play significant roles in the theory of nonlinear processes. One of the basic models for studying the nonlinear processes is the wellknown sine-Gordon model (a simple mechanical realization of which is a chain of nonlinear oscillators linked by coil springs) [8].

Another example of a strong nonlinear system playing a significant role in modern researches is hysteresis. Hysteretic behavior is typical both for the characteristics

\footnotetext{
*e-mail: melechp@yandex.ru

**e-mail: mk1150@mail.ru
}

of substances (e.g., ferroelectrics, ferromagnetics, piezoelectrics etc.), and for the dynamics of many mechanical systems (backlash, stop, etc.). In the mechanical systems hysteretic nonlinearities arise due to an aging of the material and must be taken into account at the modeling level for adequate description of the corresponding mechanical systems.

The purpose of this paper is to investigate the dynamics of oscillatory system taking into account the hysteretic coupling conditions between individual links of such a system. We consider the discrete hysteretic sine-Gordon model (or modified sine-Gordon model corresponding to the case when the links between pendulums contain hysteresis nonlinearities. On the basis of numerical modeling, the dynamics of soliton-like solutions in such a system is studied.

\section{Bouc-Wen model}

Dependencies of hysteretic type are determined by inputoutput correspondences, when the output depends not only on the instantaneous value of the input, but also on its behavior in the preceding moments of time (memory effect, or the hereditarity property).

Mathematical models of hysteresis-type phenomena are rather diverse and include both design models (backlash, stop, non-ideal relay, as well as their continuous analogues, namely the Ishlinskii and Preisach models [9]), and phenomenological models (S-converter, Duhem model, Bouc-Wen model etc. [10-13]). In this paper, we focus on the phenomenological approach based on the Bouc-Wen model for describing the hysteresis nonlinearity [13-15]. Let us recall some basic ideas of this model. 
Let us consider the equation of motion of a singledegree-of-freedom system:

$$
\mu \ddot{u}(t)+F(u, z)=f(t),
$$

where $\mu$ is the mass, $u(t)$ is the displacement, $F(u, z)$ is the restoring force and $f(t)$ is the excitation force (the overdot corresponds to the derivative with respect to time). Following the Bouc-Wen approach the restoring force is presented as (the corresponding function depends on the input and output states)

$$
F(u, z)=\alpha k u(t)+(1-\alpha) k z(t) .
$$

From this relation it follows that the restoring force $F(u, z)$ can be divided into elastic and hysteretic parts, where $k$ is the yielding stiffness, $\alpha$ is the ratio of post-yield to preyield (elastic) stiffnesses and $z(t)$ is the non-dimensional hysteretic parameter that obeys the following nonlinear differential equation with zero initial condition $(z(0)=0)$ :

$$
\dot{z}(t)=\left[A-|z(t)|^{n}(\beta+\operatorname{sign}(z(t) \dot{u}(t)) \gamma)\right] \dot{u}(t),
$$

where, $A, \beta, \gamma$ and $n$ are non-dimensional parameters controlling the behavior of the model and $\operatorname{sign}(\cdot)$ is the standard signum-function. Parameters $\beta$ and $\gamma$ control the size and shape of the hysteretic loop. Thus, such a multiparameter model describes wide class of hysteretic systems [13-15].

\section{Discrete hysteretic sine-Gordon model}

The most well-known and well-studied equations in mathematical physics are equations describing the propagation of waves in a linear medium. For a nonlinear medium with hysteretic properties, there are no ready-made methods for solution of such equations.

One of the interesting results of the analysis of wave propagation processes in nonlinear media is the existence of soliton solutions - solitary waves behaving like particles. One of the models that has a soliton solution is the sine-Gordon system. In the mechanical language this system can be presented as a chain of nonlinear pendulums with elastic torsion-tied links. Let us note here that this system has many applications, including the propagation of crystal defects and domains in ferromagnetic and ferroelectric materials, the propagation of splay waves on biological (lipid) membranes, one-dimensional model of elementary particles and propagation of magnetic flux quanta in the long Josephson junction [8].

In what follows we consider a mechanical system with hysteretic links. The physical model of such a system is shown in figure 1. It is a chain of identical pendulums strung on a string and connected by springs [16]. Pendulums oscillate transversely to the direction of the chain. The principal feature of the mechanical system under consideration is that the backlash-type hysteretic nonlinearity [17] is included in the link between two neighboring pendulums. This system is a modification of the classical mechanical sine-Gordon system and can be called hysteretic sine-Gordon system.

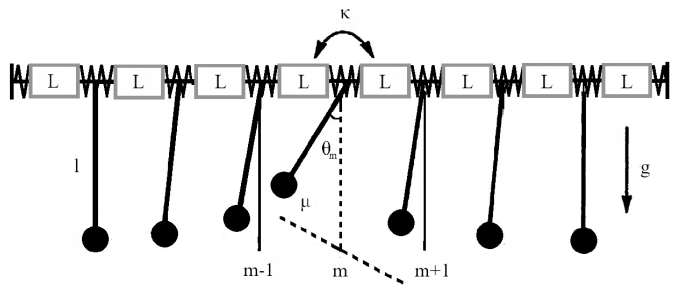

Figure 1. Discrete hysteretic sine-Gordon system

Let $\mu$ be the mass of the pendulum, $\mu l^{2}$ is the moment of inertia, $l$ is the length, and $\kappa$ is the torsion constant of the spring. When the deviation of the pendulum with number $m$ from the equilibrium point by an angle $\theta_{m}$ takes place, the gravitational force moment $-\mu g l \sin \theta_{m}$ acts on the pendulum alongside the torsional moment acting on the side of adjacent springs $-\kappa\left(\theta_{m}-\theta_{m-1}\right)+\kappa\left(\theta_{m+1}-\theta_{m}\right)$. Since the hysteretic nonlinearity is included in the system, the equation of motion can be presented as:

$$
\left\{\begin{array}{l}
\mu l^{2} \ddot{\theta}_{m}=-\mu g l \sin \theta_{m}+\omega_{m}^{\text {left }}+\omega_{m}^{\text {right }}, \\
\omega_{m}^{\text {left }}=L\left[\omega_{m}^{\text {left }}\left(t_{0}\right) ; y_{m}^{\text {left }}\left(t_{0}\right)\right] y_{m}^{\text {left }}(t), \\
y_{m}^{\text {left }}=-\kappa\left(\theta_{m}-\theta_{m-1}\right), \\
\omega_{m}^{\text {right }}=L\left[\omega_{m}^{\text {right }}\left(t_{0}\right) ; y_{m}^{\text {right }}\left(t_{0}\right)\right] y_{m}^{\text {right }}(t), \\
y_{m}^{\text {right }}=\kappa\left(\theta_{m+1}-\theta_{m}\right),
\end{array}\right.
$$

where the time-dependent outputs $\omega_{m}^{\text {left }}, \omega_{m}^{\text {right }}$ and inputs $y_{m}^{\text {left }}, y_{m}^{\text {right }}$ (these inputs are the corresponding moments affecting single pendulum from the left and right sides relative to neighbor pendula respectively) are the corresponding outputs and inputs for the physically realizable converter $L[\cdot]$ in the frame of Krasnosel'skii and Pokrovskii approach [9], and $\omega_{m}\left(t_{0}\right), y_{m}\left(t_{0}\right)$ are the corresponding initial states (output and input, respectively) of the converter.

\section{Numerical simulation}

It is known that the operator interpretation of the hysteretic nonlinearity (as presented in system (4) implies the nonsmoothness of the corresponding operator. Therefore, in our numerical simulation we use the approach to hysteresis based on the Bouc-Wen model. In this case, the discrete 
hysteretic sine-Gordon system takes the following form:

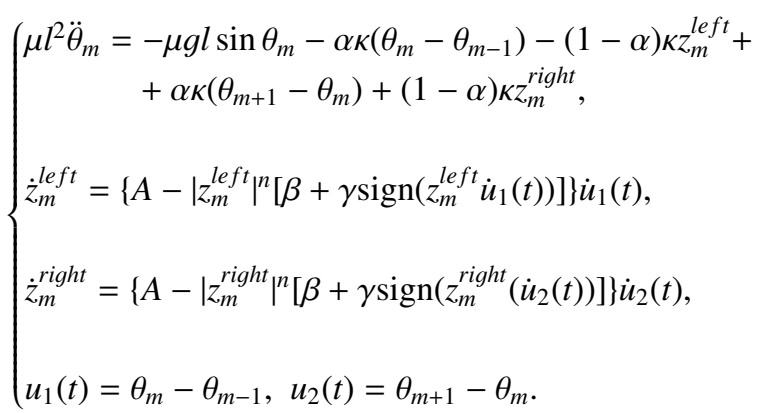

We performed the numerical simulation of the dynamics of the mechanical system described by equations (5) using the 4-th order Runge-Kutta method. The chain has a finite length $m=100$ (we recall that we consider a discrete system), and its ends are fixed. The initial conditions for pendulums

$$
\theta_{1}\left(t_{0}\right), \dot{\theta}_{1}\left(t_{0}\right), \ldots, \theta_{m-1}\left(t_{0}\right), \dot{\theta}_{m-1}\left(t_{0}\right), \theta_{m}\left(t_{0}\right), \dot{\theta}_{m}\left(t_{0}\right)
$$

generate a family of solitonic solutions moving with different velocities along the $(m, t)$-plane with reflection at the ends of the chain $(m=1, m=100)$. For the parameters of the hysterestic blocks formalized by means of the Bouc-Wen model, $\dot{z}_{m}^{l e f t}\left(t_{0}\right), \dot{z}_{m}^{\text {right }}\left(t_{0}\right)$, the initial conditions are zero by default.

In order to study the influence of hysteresis bonds in the system, we consider the case in which the vibrations of 25th $\left(\theta_{25}\left(t_{0}\right)=2 \pi, \dot{\theta}_{25}\left(t_{0}\right)=0\right)$ and 75 th $\left(\theta_{75}\left(t_{0}\right)=\right.$ $\pi, \dot{\theta}_{75}\left(t_{0}\right)=0$ ) pendulums are excited with the corresponding initial conditions. Under these initial conditions, the oscillations of the corresponding components are excited in the chain (fig. 2 (top panel)). In the case when the hysteresis in the links is taken into account (fig. 2 (bottom panel)), spatial localization of oscillations is observed.

Let us consider in more detail the evolution of the states of the components of the chain $(\theta(t), \dot{\theta}(t))$ in the neighborhood of 25 th pendulum. Figure 3 shows the phase portraits for 24th, 25th, and 26s pendulums, respectively together with corresponding hysteretic loops (such loops are obtained as a numerical solution to equation 3 of the Bouc-Wen model). As follows from this figure, in the absence of hysteretic links $(\alpha=1)$ the dynamics of pendulums demonstrates a complex oscillatory structure. However, in the presence of hysteresis in the links ( $\alpha=0.5, \beta=0.1, \gamma=0.9$ ), the dynamics in the neighborhood of the 25 th pendulum is regularized and the stable limit cycle can be seen.

Also, we investigated the influence of the hysteretic blocks in the links between pendulums by using the methods of spectral analysis. We performed the Fourier transform for the 25th pendulum in the presence of hysteretic block ( $\alpha=0.5, \beta=0.1, \gamma=0.9)$ and without $(\alpha=1)$ it. The corresponding results are shown in figure 4.

As it follows from the results presented in this figure, the oscillation spectrum changes after inclusion of hysteretic bonds. Thus we can conclude that the hysteresis in such a system plays a role of a "filter" that quenches frequencies corresponding to small-amplitude oscillations and releases the main frequency.
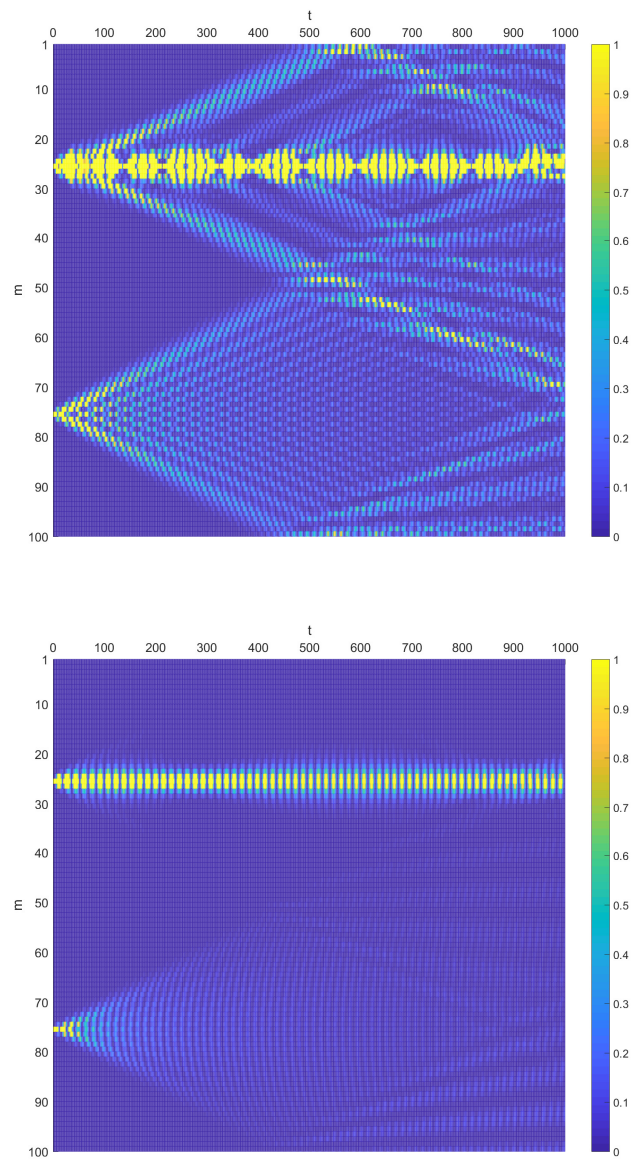

Figure 2. Simulation of the dynamics of localized (soliton-like) oscillations of pendulums in a chain without hysteresis $(\alpha=1$, top panel) and with hysteresis $(\alpha=0.75, \beta=0.1, \gamma=0.9$, bottom panel) in the links

\section{Conclusions}

In this paper we studied the dynamics of an oscillatory system with many degrees of freedom under conditions of hysteretic blocks in the coupling between the individual parts of the system. The system under consideration can be classified as a discrete hysteretic sine-Gordon model (the connections between the pendulums contain a hysteretic nonlinearity). The hysteretic nonlinearity was formalized by means of the Bouc-Wen model which allows a fairly simple numerical realization of the solution to the system of equations corresponding to the mechanical system under consideration. On the basis of numerical simulations, the dynamics of the solitonic solution for this system was studied taking into account the hysteretic nature of the coupling. The results of numerical simulation demonstrate the regularizing role of hysteresis bonds in the character of oscillatory motions in this mechanical system. The filtering properties of hysteretic bonds are inferred from the spectral analysis of the oscillatory motions of individual components of the system.

This work is supported by the RFBR (Grants 16-08-00312-a, 1701-00251-a, and 18-08-00053-a) 

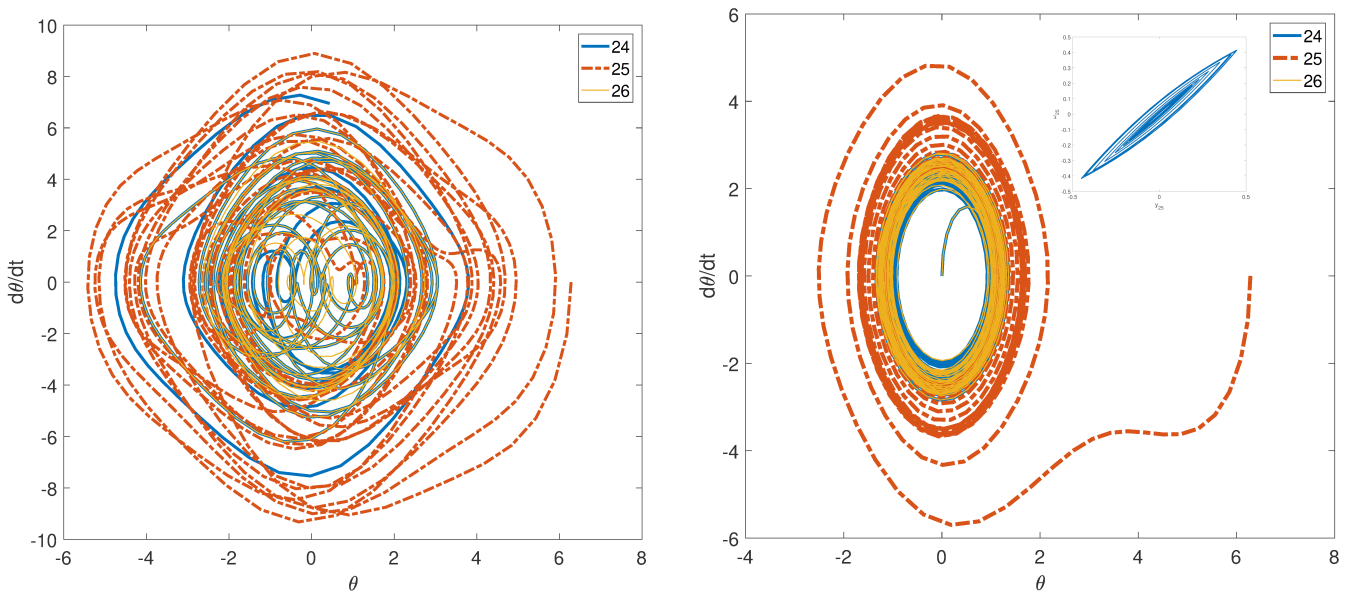

Figure 3. Phase portraits of 24 th, 25 th, $26 \mathrm{~s}$ pendulums without hysteresis (top panel) and with hysteresis (bottom panel) in the links. The inset (bottom panel) shows the corresponding hysteretic loop obtained as a solution to eq. (3).
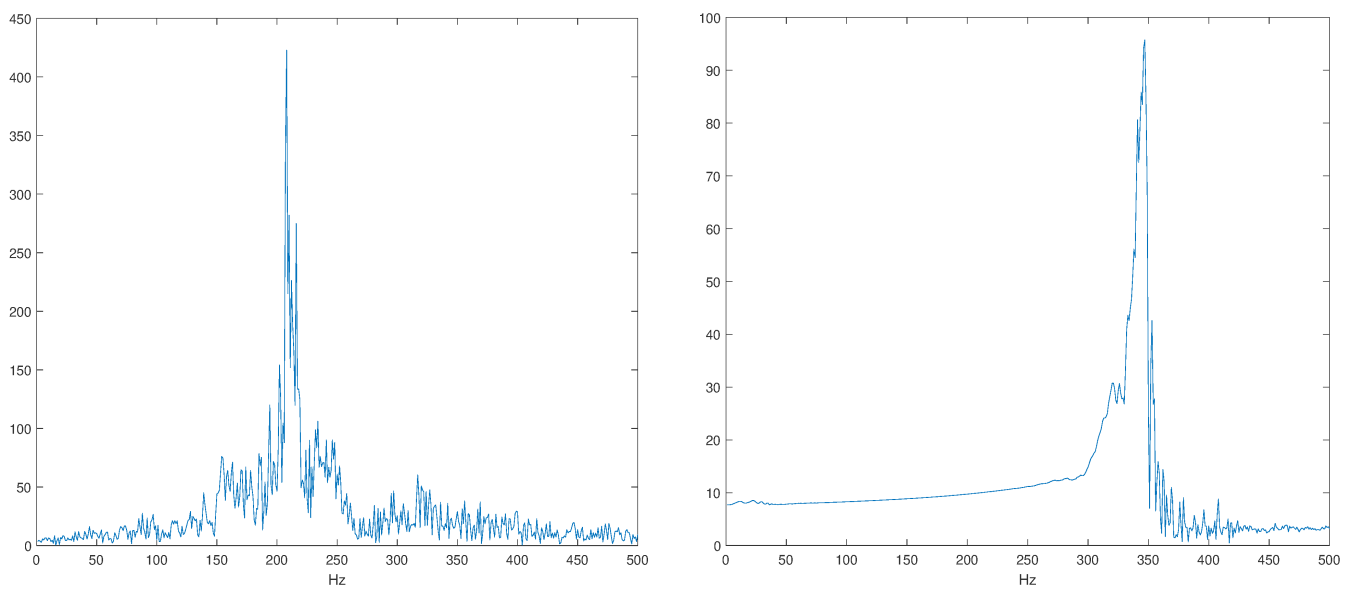

Figure 4. The oscillation spectrum of the 25th pendulum without hysteresis (top panel) and with hysteresis (bottom panel) in the link

\section{References}

[1] Lichtenberg A J, Livi R, Pettini M, Ruffo S, Lecture Notes in Physics, 728 21-121 (2008)

[2] Torre C G, Foundations of Wave Phenomena $1927-$ 38 (2014)

[3] Sieber J, Kalmar-Nagy T, Phys. Rev. E 84 016227(16) (2011)

[4] Semenov M E, Solovyov A M, Popov M A, Meleshenko P A, Arch. Appl. Mech., 87 DOI: https://doi.org/10.1007/s00419-017-1323-0 (2017)

[5] Scott A C, Active and nonlinear wave propagation in electronics (Wiley-Interscience, New York, 1970) $326 \mathrm{p}$

[6] Trubetskov D I, Rozhnev A G, Lineynyye kolebaniya $i$ volny (Fizmatlit, Moscow, 2001) $419 \mathrm{p}$

[7] Haken H, Quantum field theory of solids: an introduction (North-Holland, Amsterdam, New York, 1976) $330 \mathrm{p}$

[8] Scott A C, Nonlinear Science. Emergence and Dynamics of Coherent Structures (Oxford, Oxford Uni- versity Prees, 1999) $474 \mathrm{p}$

[9] Krasnosel'skii M A, Pokrovskii A V, Systems with Hysteresis (Berlin, Springer-Verlag, 1989) $410 \mathrm{p}$

[10] Bouc R, Proceedings of the Fourth Conference on Nonlinear Oscillations (Prague, Czechoslovakia) 315-321 (1967)

[11] Bouc R, Acustica 21 16-25 (1971)

[12] Wen Y K, Journal of the Engineering Mechanics Division 102 (2) 246-263 (1976)

[13] Ikhouane F, Rodellar J, Nonlinear Dynamics, 42 6378 (2005)

[14] Charalampakis A E, Archive of Applied Mechanics 85 549-555 (2015)

[15] Solovyov A M, Semenov M E, Meleshenko P A, Barsukov A I, Procedia Engineering 201 549-555 (2017)

[16] Scott A C, Am. J. Phys 37 52-61 (1969)

[17] Solovyov A M, Semenov M E, Meleshenko P A, Reshetova O O, Popov M A, Kabulova E G Procedia Engineering 201 578-583 (2017) 